\title{
Biopsia transrectal ecodirigida de la próstata: estado del arte y guía práctica de consejos y trucos
}

\section{Transrectal Ultrasound Biopsy of the Prostate: State of the Art and Practical Guide of Tip and Tricks}

\author{
Julián Chavarriaga Soto ${ }^{1}$ María Antonia Ocampo ${ }^{2}$ Andrés Felipe Gutiérrez ${ }^{3}$
}

${ }^{1}$ Residente de Urología, Pontificia Universidad Javeriana, Bogotá, Colombia
2 Estudiante de Medicina, Universidad del Rosario, Bogotá, Colombia
${ }^{3}$ Departamento de Urología, Hospital Universitario San Ignacio, Profesor
Ad Honorem Pontificia Universidad Javeriana, Bogotá, Colombia

Address for correspondence María Antonia Ocampo, Estudiante de Medicina, XII Semestre, Universidad del Rosario, Bogotá, Colombia (e-mail: ocampog.maria@gmail.com).

Urol Colomb 2019;28:130-141.

\section{Resumen \\ Palabras Clave \\ - cáncer de próstata \\ - próstata \\ - aspiración por aguja fina ecodirigida \\ - ecografía \\ - biopsia}

Introducción y Objetivos La biopsia transrectal de la próstata (BTRP), fue propuesta por primera vez en 1937 y hasta 1981 se realizó la primera biopsia ecodirigida, actualmente es la vía de acceso a la próstata más utilizada por su fácil curva de aprendizaje como por el alto rendimiento diagnóstico, sin embargo, en el Reino Unido, el $68 \%$ de los urólogos no realizan BTRP porque consideran que no han recibido suficiente entrenamiento. El objetivo de este estudio es describir las diferentes técnicas utilizadas en la actualidad, las complicaciones del procedimiento y aportar una guía de consejos y trucos implementada en varios centros de referencia a la hora de realizar una BTRP para prevenir complicaciones, mejorar el desempeño de la prueba y del urólogo y estandarizar el método de toma de la BTRP.

Materiales y Métodos Realizamos una búsqueda en las bases de datos de PubMed, MEDLINE, SciELO utilizando las palabras claves "Transrectal ultrasound biopsy of the prostate" "tips and tricks" "Transperineal biopsy of the prostate" "Magnetic resonance imaging targeted biopsy" "MRI/US fusion biopsy", basados en la literatura y en la experiencia de los autores de más de 1100 biopsias anuales entre los diferentes centros de referencia. Brindamos una guía práctica de consejos y trucos para facilitar el desempeño del urólogo en la BTRP.

Resultados La biopsia transrectal de próstata ecodirigida continúa siendo la primera opción para el abordaje diagnóstico del paciente con sospecha clínica de cáncer de próstata, es de gran importancia estandarizar el esquema de toma de la biopsia, y en nuestro caso recomendamos utilizar un esquema de 12 cores, definir la profilaxis antibióticas y la duración del tratamiento, y el uso de analgesia o anestesia local. Presentamos los consejos y trucos que hemos utilizado en nuestra práctica clínica en varios centros de referencia.

\section{Abstract}

Introduction Transrectal biopsy of the prostate was described for the first time in 1937, and it was not until 1981 the first transrectal biopsy of the prostate was done

received

March 21, 2018

accepted

May 25, 2018
DOI https://doi.org/ $10.1055 / \mathrm{s}-0038-1661364$. ISSN 0120-789X. eISSN 2027-0119.
Copyright (c) 2019, Sociedad Colombiana License terms de Urología. Publicado por Thieme Revinter Publicações Ltda., Rio de Janeiro, Brazil. Todos los derechos reservados.

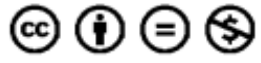




\section{Keywords}

- cancer of the prostate

- prostate

- endoscopic ultrasound guided fine needle aspiration

- ultrasound

- biopsy using transrectal ultrasound to guide the procedure. Nowadays it is the most popular technique to obtain prostatic tissue when suspecting adenocarcinoma of the prostate, this due to its easy learning curve and to its excellent diagnostic performance. Up to $68 \%$ of urologist in the UK do not do prostate biopsy arguing they have not received enough training The aim of this article is to describe the different techniques, the physics of ultrasonography and a practical guide of tips and tricks from a center where we performed up to 1100 transrectal ultrasound biopsies annually, aiming to improve the diagnostic performance and lower the complicacion rates of the biopsy.

Methods We performed a search in PubMed, MEDLINE, SciELO using the keywords "Transrectal ultrasound biopsy of the prostate" "tips and tricks" "Transperineal biopsy of the prostate" "Magnetic resonance imaging targeted biopsy" "MRI/US fusion biopsy," and base on the literature review and our experience of more than a thousand biopsies annually we wrote this article.

Results TRUS biopsy of the prostate continue to be the first choice in the urologist armamentarium to diagnose the patient with clinical suspicion of prostate cáncer, with a low complication rate, a good diagnostic performance and an easy learning curve. It is necessary to standardized the procedure, perform a doble sextant biopsy, define the best antibiotic prophylaxis, the technique for anesthesia/analgesia. Here we present our practical guide of tips and tricks.

\section{Introducción}

El cáncer no cutáneo más diagnosticado en el mundo es el cáncer de próstata, con un estimado de 903,500 nuevos casos de cáncer de próstata en el mundo anualmente; ${ }^{1,2}$ se ha descrito que un hombre norteamericano tiene una probabilidad de ser diagnosticado con esa enfermedad de uno en siete y una probabilidad de uno en treinta de morir por la misma. ${ }^{3}$ Desde la década de los $80 \mathrm{y}$ con el advenimiento del tamizaje con Antigeno prostático especifico (PSA), se establecieron puntos de corte para determinar la necesidad de BTRP para la confirmación histopatológica del diagnóstico de cáncer, eso ha permitido que se disminuya la mortalidad cáncer-específica y se diagnostiquen más casos de cáncer de próstata localizado y menos casos de enfermedad metastásica. ${ }^{1-6}$

La decisión de realizar una BTRP debe ser una decisión informada tomada en conjunto con el paciente, se debe explicar las posibles complicaciones, las medidas que se tomarán para evitarlas y el desempeño de la prueba diagnóstica, en este caso la BTRP. Las indicaciones aceptadas actualmente son: Tacto rectal sospechoso con un riesgo de cáncer de 5\% al 30\%, en el caso de diagnóstico incidental de cáncer de próstata en tejido de resección transuretral de la próstata (RTUP), síntomas o hallazgos al examen físico o imagenológicos que sugieran enfermedad metastásica, elevación del PSA, teniendo en cuenta los puntos de corte para cada grupo etario y factores de riesgo específicos de cada paciente. ${ }^{7-9}$

Los pioneros de la BTRP fueron Grabstald y Elliot (1953), Graham (1958) y Daves (1961), sin embargo la primera BTRP se le atribuye a Astraldi en 1937; la técnica era dígitodirigida, y con ella, Franzen inventa un dispositivo para facilitar el procedimiento que utilizaba un anillo en el dedo índice por el que se introducía la aguja y permitía la ubicación precisa de la aguja sobre la lesión, que se denominó la aguja y guía de Franzen y fue Williams quien, utilizando ese dispositivo, publicó los primeros resultados satisfactorios de BTRP en $1971 .^{10-13}$

En 1963 Takashi y Ouchi reportaron el uso de ecografía transrectal para evaluar la próstata, sin embargo la calidad de la imagen era muy pobre y no tenía utilidad clínica, hasta 1967 que Watanabe obtuvo las primeras imágenes de buena calidad para evaluar clínicamente la próstata con un transductor de $3.5 \mathrm{MHz}$; pasaron más de 13 años hasta que en 1980 con las mejoras tecnológicas de los transductores ecográficos, se realiza por primera vez una BTRP ecodirigida, en 1989 Hodge publicó el artículo que sería el hito de las BTRP modernas, en el que él describía la BTRP en sextantes con la que lograba detectar 9\% más cánceres que con la BTRP dirigida, por lo cual se convirtió en el patrón de oro por muchos años. Stamey en 1995 modificó la técnica en sextantes y tomó biopsias en sextantes que eran laterales a la línea medio-sagital. ${ }^{14,15}$ La técnica más aceptada en la actualidad fue descrita por Levine, quien describió la técnica de doce-cilindros 0 doble sextante. ${ }^{16}$ Posteriormente, fue Stewart el primero en hablar de biopsia por saturación en el 2001 en el que 20 o más cilindros se obtenían de manera sistemática y solo se deben ofrecer en el escenario de la re-biopsia. ${ }^{12-14}$

El objetivo de este trabajo es presentar una revisión detallada de la literatura sobre BTRP, técnicas de biopsia, complicaciones y como prevenirlas, re-biopsia y biopsia del lecho prostático en la actualidad; y aportar una guía practica de consejos y trucos desde nuestra experiencia personal con mas de 3.000 biopsias. 


\section{Materiales y Métodos}

Realizamos una búsqueda en las bases de datos PubMed, MEDLINE y SciELO utilizando las palabras claves "Transrectal ultrasound biopsy of the prostate" "tips and tricks" "Transperineal biopsy of the prostate" "Magnetic resonance imaging targeted biopsy" "MRI/US fusion biopsy". Basados en los resultados encontrados en la literatura y en la experiencia clínica de los autores con más de 1100 casos por año, describimos los pasos que se deben seguir al realizar una BTRP, y aportamos los trucos y consejos que consideramos pueden facilitarle al urólogo la realización de una BTRP, optimizando así el desempeño de la prueba y disminuyendo la tasa de complicaciones.

\section{Resultados}

La revisión se divide en cuatro grupos:

1. Técnicas para BTRP: Esquemas, instrumentos y volumetría prostática.

2. Complicaciones de la BTRP ¿Cómo prevenirlas?

3. Re-biopsia: Biopsia por Saturación, biopsia guiada por RNMmp y biopsia del lecho prostático.

4. Consejos y trucos en nuestra práctica clínica para realizar una BTRP.

\section{Técnicas para BTRP: Esquemas, instrumentos y volumetría prostática}

En la actualidad la BTRP se hace dirigida por ultrasonografia (US), esa modalidad de imagen evalúa en tiempo real los tejidos sin exposición a radiación ionizante; funciona mediante la interacción entre las ondas de sonido y los tejidos, esas ondas se producen a través de la aplicación de salvas cortas de corriente eléctrica alternada, a una serie de cristales alojados en el transductor, así el efecto piezoeléctrico genera una onda mecánica que se transmite a través de un medio de acoplamiento (Gel) al recto, el transductor actúa a la misma vez como receptor y "escucha" la onda de sonido que regresa y convierte la energía mecánica en eléctrica, amplifica la señal y le asigna un valor en escala de grises proporcional a la fuerza de la señal que regresa al transductor. La frecuencia para las ondas de sonido oscila entre 3,5-12 MHz. En ecografía transrectal de la próstata, se utilizan transductores de $5 \mathrm{MHz}$ en adelante ya que ella se encuentra justo anterior al recto y no requerimos mayor penetración de las ondas. Sin embargo, se debe tener en cuenta que existe un efecto opuesto entre penetrancia y resolución. ${ }^{15,17-19}$

Se pueden ajustar diferentes variables del ecógrafo para mejorar la calidad de la imagen en la escala de grises, esas son el poder de salida que es el poder de emisión del sonido en decibeles, así a mayor poder de salida mayor es el reflejo de la señal que se capta por el transductor y le da más brillo a la imagen, la ganancia del receptor amplifica selectivamente la señal de retorno logrando mayor o menor brillo de la imagen, la compensación tiempo ganancia (T-G) que permite una amplificación más refinada del sonido, estableciendo así, diferentes distancias de profundidad con la manipulación de la superficie del transductor y la zona focal que es el punto focal óptimo del transductor, que debe establecerse en el borde más distal de la glándula o el punto más distal de interés. ${ }^{16-19}$

Al evaluar la próstata por USTR debemos estar familiarizados con la anatomía usual; la próstata se divide en cinco zonas: estroma fibromuscular anterior (EFMA), zona peri-uretral (ZPU), zona transicional (ZT), zona central (ZC) y zona periférica (ZP); de esta última surgen el $70 \%$ de los cánceres de próstata, desafortunadamente esas regiones no se pueden diferenciar ecográficamente con facilidad. La próstata es una estructura retroperitoneal, se encuentra anterior al recto, entre el cuello vesical y el diafragma urogenital que resembla un anillo fibroso y contiene la uretra, entre la glándula y el recto está la fascia de Denonvilliérs, y lateral está la fascia del músculo elevador del ano y el músculo obturador interno, eso ubica a la próstata en una posición ideal para la evaluación por USTR ${ }^{18,19}$ (- Fig. 1).

La BTRP debe ir precedida de un tacto rectal y posteriormente una evaluación ecográfica de la próstata, ella se debe realizar con un transductor endorectal en los planos transverso y sagital, se debe comenzar en la base y extenderse hacia el ápex, describir las características y localización de las lesiones, evaluar la cápsula, chequeo de simetría del paquete neurovascular en la cara posterolateral de la próstata, presencia de calcificaciones, anormalidad de los contornos de la próstata y se debe evaluar las vesículas seminales describiendo su ecogenicidad, tamaño, posición, simétria y si hay compromiso del ángulo vesículo-prostático. Como parte de esa evaluación, también es importante calcular la volumetría prostática, se han descrito múltiples fórmulas para hacerlo, sin embargo, convencionalmente la más aceptada es quizás la elipsoidal con el pretexto de que la próstata imita una elipse al hacer una reconstrucción tridimensional de la misma; también se ha descrito que la próstata puede imitar una esfera, un esferoide y puede tener forma de huevo "egg-shaped" o de bala "bulletshaped", todas esas fórmulas predicen el volumen prostático con

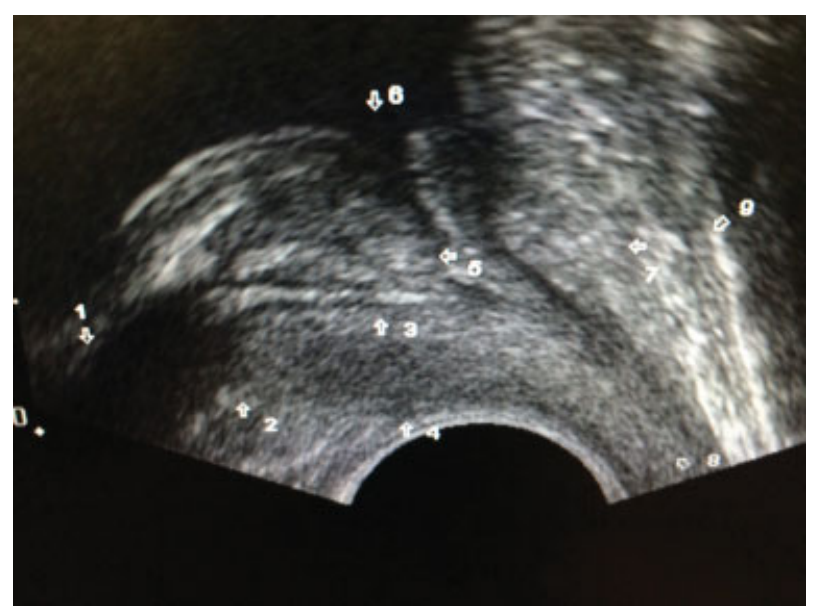

Fig. 1 En esta imagen podemos ver la anatomia usual de la prostata en USTR, utilizando un transductor End-Fire en una vista longitudinal. 1. Vesícula seminal. 2. Ángulo vesículo-prostático. 3. Conducto eyaculador. 4. Zona periférica. 5. Zona transicional. 6. Cuello vesical. 7. Estroma fibromuscular anterior. 8. Ápex de la próstata. 9. Plexo venoso dorsal del pene. (Original). 


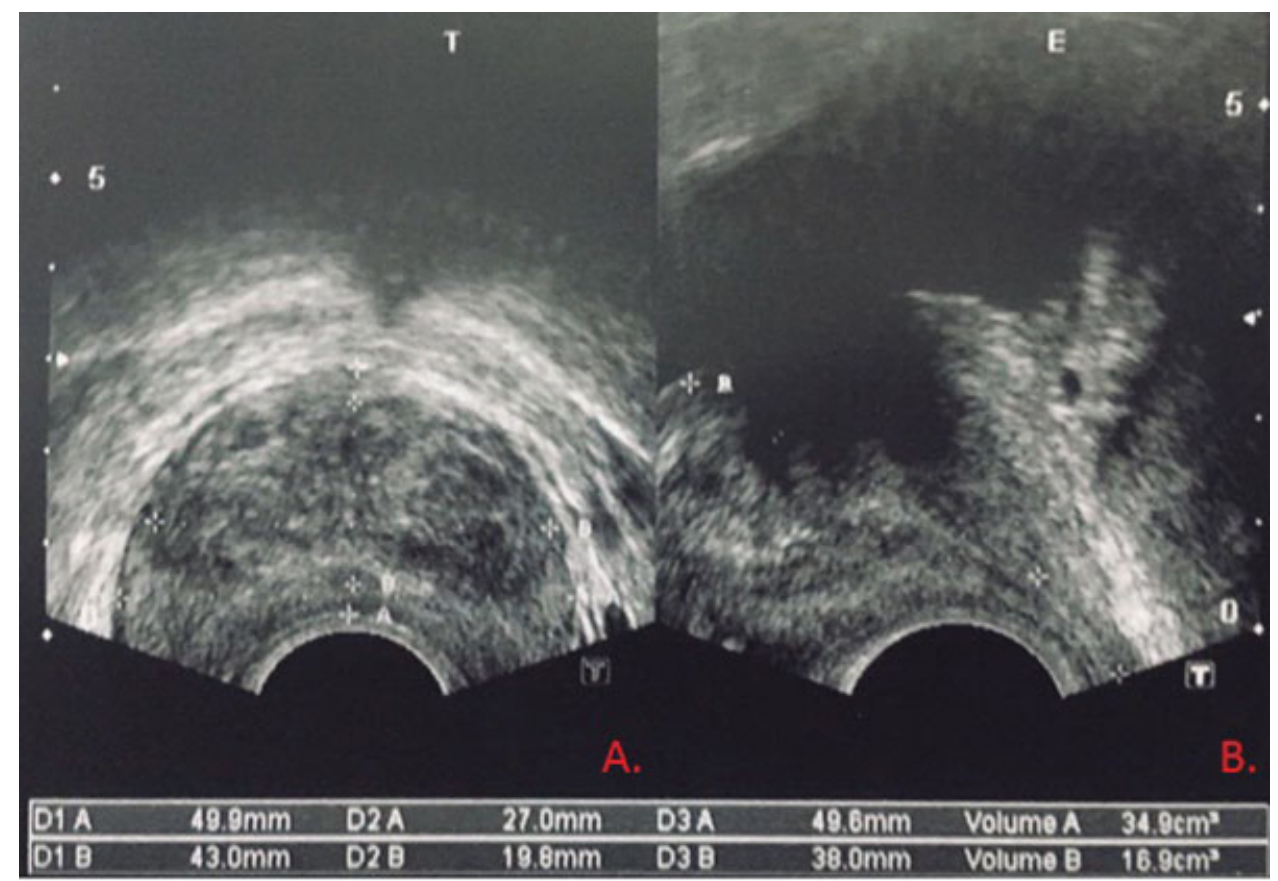

Fig. 2 Cálculo de la volumetría prostática utilizando la fórmula del elipsoide. En el plano transverso (A.) se toman las medidas de ancho y largo y en el plano longitudinal (B.) se toma la medida de altura. (Original).

coeficientes de correlación $>0,9$ al compararlo con el peso del espécimen de prostatectomía radical, asumiendo que $1 \mathrm{~cm}^{3}=1$ gramo. ${ }^{18,19}$ Para calcular el volumen de la próstata se requiere la medición en los planos axial y sagital de las tres dimensiones de la glándula: largo, ancho máximo y altura máxima ortogonal ${ }^{20}$ (-Fig. 2). Para calcular el volumen elipsoidal, que es la forma más aceptada actualmente se utiliza la fórmula:

\section{Volumen Elipsoidal: Largo x Ancho x Altura x $\frac{\Pi}{6}$}

Dentro de los hallazgos normales en la USTR de la próstata se pueden encontrar lesiones quísticas que normalmente tienen una pared delgada, son anecoicas y muestran un realce acústico, ellos usualmente son de origen congénito y pueden derivar de remanentes mullerianos o estructuras wolffianas, o adquiridos, y no tienen significado clínico; también se pueden identificar cuerpos amiláceos entre la ZP y ZT que son calcificaciones benignas por el envejecimiento. No debemos olvidar que la apariencia ecográfica de la próstata puede cambiar después de radioterapia, terapia de supresión androgénica, resección transuretral de la próstata (RTUP) y prostatectomía radical. ${ }^{18,19}$ Las lesiones hipoecoicas en USTR por mucho tiempo se consideraron patognomónicas del cáncer de prostata, posteriormente se demostró que hasta el 39\% de las lesiones cancerígenas pueden ser iso-ecoicas y hasta $1 \%$ hiperecoicas, por esa razón, se dice que la USTR tiene una precisión diagnosticada limitada para evaluar el cáncer de próstata ya que posee una baja tasa de verdaderos negativos y una tasa alta de falsos positivos. Los signos ecográficos altamente sugestivos de cáncer son áreas hipoecoicas en la zona periférica especialmente nodulares con alteración de la ecogenicidad alrededor del nódulo, irregularidad del contorno de la cápsula. Se debe sospechar extensión extra-prostática (cT3), cuando haya pérdida focal de la apariencia típica blanquecina brillante de la grasa periprostatica, un abultamiento irregular hipoecoico de la cápsula, asimetría de los paquetes neurovasculares y pérdida del ángulo agudo que se forma entre las vesículas seminales y la próstata. Esos hallazgos son más fáciles de apreciar en un plano transversal.

La BTRP no significa tomar un número de muestras al azar sino que se debe seguir un esquema sistemático para asegurar una tasa de detección de cáncer adecuada. Vale la pena resaltar que el primer esquema que se consideró el patrón de oro fue el esquema en sextantes que propone tomar un cilindro de la base, de la porción media de la glándula y del ápex bilateral, en la línea medio-sagital. Con ese esquema se dejaba de diagnosticar el 30\% de los cánceres de próstata. Posteriormente Levine publicó su estudio en 1998, utilizando el esquema de doble sextante que seguía el esquema de la biopsia por sextante clásica pero en la misma intervención tomaba seis muestras en la zona lateral de la glándula aumentando así la detección de la enfermedad en un $10 \%$, por lo que hoy en día ese esquema es quizás el más ampliamente aceptado y es el recomendado por las guías de práctica clínica ${ }^{15,16}$ (- Fig. 3 ).

Hay dos tipos de transductores o sondas ultrasonográficas, que son de disparo lateral "side-fire" o disparo terminal "endfire", la diferencia radica en que el transductor "side-fire" permite una trayectoria de biopsia limitada en el eje longitudinal (Base a ápex), dado que a pesar de que permite visión en eje transversal y sagital, para obtener el eje transversal, el transductor debe ubicarse posterior a la glándula, así cuando la aguja pasa la sonda en un eje craneocaudal se dificulta biopsiar la porción anterior y el ápex de la próstata, las cuales solo se pueden lograr con un torque exagerado del equipo, adicionalmente debe evitarse atravesar el recto por debajo de la línea dentada a la hora de realizar una biopsia apical ya que puede resultar doloroso y 


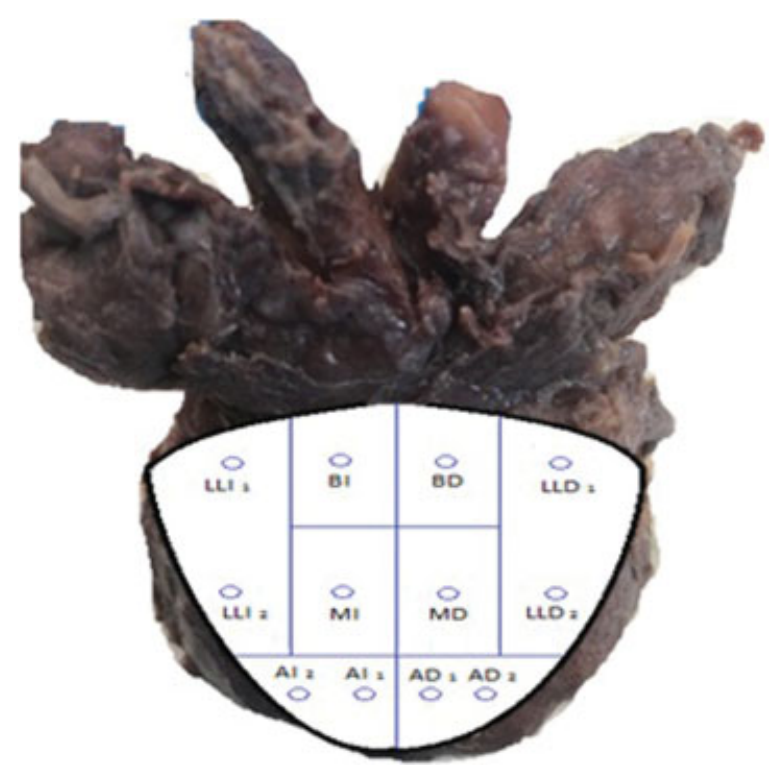

Fig. 3 Esquema de BTRP en doble sextante. LLI: Lóbulo lateral izquierdo, Al: ápice izquierdo, MI: medial izquierdo, Bl: base izquierda, LLD: Lóbulo lateral derecho, $A D$ : ápice derecho, MD: medial derecho, $B D$ : base derecha. (Original).

molesto para el paciente, mientras que el transductor "endfire" permite una trayectoria transversal o en un eje AP y la forma de la punta del transductor es curva, lo que mejora la flexibilidad a la hora de maniobrar el equipo para tomar la biopsia y permite tomar más fácilmente biopsias de la región anterior y apical. Chin y col. compararon la BTRP con el transductor "side-fire" en 1224 pacientes o "end-fire" en 1550 pacientes y encontraron que la tasa de detección de cáncer era mayor en los pacientes estudiados con transductor "end fire" 45,8 vs $38,5 \%$ con una p estadísticamente significativa, sin embargo, ese estudio tiene una limitación y es que es un estudio observacional, no aleatorizado. ${ }^{18,21,22}$

Los transductores endorectales están disponibles en frecuencias de 6 a $10 \mathrm{MHz}$ y permiten un ángulo de mapeo (visión), aproximadamente de 180 grados, lo que permite la visualización simultánea de toda la glándula en los planos sagital y transversal. Con respecto a la manipulación del transductor, varía de acuerdo al plano sagital y transversal. En el plano sagital hay dos métodos, el primero, es rotar el transductor en el sentido horario que nos muestra imágenes del lóbulo izquierdo, y al girarlas al contrario del sentido horario nos muestra imágenes del lóbulo derecho; el otro método es en decúbito lateral derecho, se debe angular el mango del transductor hacia el piso (lóbulo derecho) o hacia el techo (lóbulo izquierdo). Esa técnica simula los movimientos que se realizan al vascular el cistoscopio por lo que los urólogos se encuentran más familiarizados con ella. Para obtener un plano transversal se debe angular el transductor hacia la derecha o la izquierda, tomando como fulcro el esfínter anal externo, entonces al angularlo hacia el piso, produce imágenes cefálicas y hacia el techo produce imágenes caudales. ${ }^{18}$

Para realizar la BTRP se utiliza una pistola de biopsia que puede ser desechable o reutilizable. A parte del costo no hay ninguna ventaja sobre la una o la otra, la pistola se pasa por el anillo guia que se encuentra adherido al transductor endorectal, esos pueden ser guías metálicas o plásticas, las metálicas se fijan con mayor seguridad, pero con la desventaja de que se deben esterilizar para cada procedimiento; se utiliza una aguja por paciente que usualmente es de 18 Gauge, al montar la pistola sobre el transductor se avanza la aguja $0,5 \mathrm{~cm}$ y se toma una muestra (cilindro) que son de 1,5-2 cm; el tejido que se obtiene se extiende hasta $0,5 \mathrm{~cm}$ más allá del área biopsiada, por lo que la recomendación es que al biopsiar la ZP se debe posicionar la punta de la aguja hasta $0,5 \mathrm{~cm}$ de la cápsula para asegurarse que la biopsia se está tomando de esa zona $^{19,23,24}$ (-Fig. 4).

\section{Complicaciones de la BTRP ¿Cómo prevenirlas?}

La BTRP se ha establecido como el estándar de cuidado en los pacientes con sospecha clínica de cáncer de próstata, se realizan entre 1 y 1,3 millones de biopsias anualmente en Estados Unidos. El hecho de obtener el tejido por vía transrectal, se clasifica como un procedimiento contaminado dada la localización del transductor y el contacto de la aguja en su trayectoria con heces y flora bacteriana rectal. Se han publicado guías de práctica clínica, como la de la Asociación Canadiense de Urología (CUA $)^{25}$ y políticas de buena práctica para BTRP (AUA) 26,27 con el objetivo de disminuir las complicaciones relacionadas con ese procedimiento. En los últimos 10 años, la tasa de complicaciones ha aumentado;28,29 en el estudio de Nam y col., observaron un aumento del $1 \%$ al $4,1 \%$ en la tasa de ingreso al hospital por complicaciones derivadas de la BTRP 30 días después de la intervención entre 1996 y 2005, al evaluar 75.190 pacientes sometidos a ese procedimiento, sin embargo, a pesar de aumentar la tasa de complicaciones y del ingreso al hospital, la mortalidad no aumentó, con una mortalidad a 30 días del $0,09 \% .{ }^{30}$ Ese aumento exponencial en el número de complicaciones, se puede explicar por el incremento en la prevalencia de microorganismos resistentes a las fluoro-quinolonas y de bacterias productoras de betalactamasas de espectro extendido (BLEE). Esa complicación, reviste de gran importancia, dado que el $72 \%$ de los ingresos fueron por alguna causa infecciosa, lo que motiva a diseñar estrategias para prevenir las complicaciones infecciosas y que permitan el uso razonable de antibióticos para la profilaxis de la BTRP. ${ }^{18}$

En la serie más grande de complicaciones en BTRP que incluyó 1875 pacientes, el 6,6\% presentó una complicación, fueron ellas: prostatitis (3,8\%), epididimitis $(0,2 \%)$, retención urinaria aguda $(2,1 \%)$ que como factores predisponentes se encuentran volumetrías prostáticas elevadas e IPSS moderado-severo, rectorragia $(0,2 \%)$, hematuria $(1,9 \%), \mathrm{y}$ sepsis (0,05\%), síncope vasovagal (0,05\%). A pesar de que esa serie reportó una incidencia muy baja de síncope vasovagal, otras series (Djavan y col.) han reportado una incidencia de hasta un $1,4 \%-5,3 \%$, por lo que debemos estar familiarizados con ese fenómeno y conocer que en este caso, se debe suspender el procedimiento, colocar al paciente en posición de trendelenburg e iniciar líquidos endovenosos. ${ }^{18,29,31}$

Actualmente, la incidencia de infección relacionada con la biopsia, oscila entre 2 y $6 \%$ y debe ser una premisa, en que en 

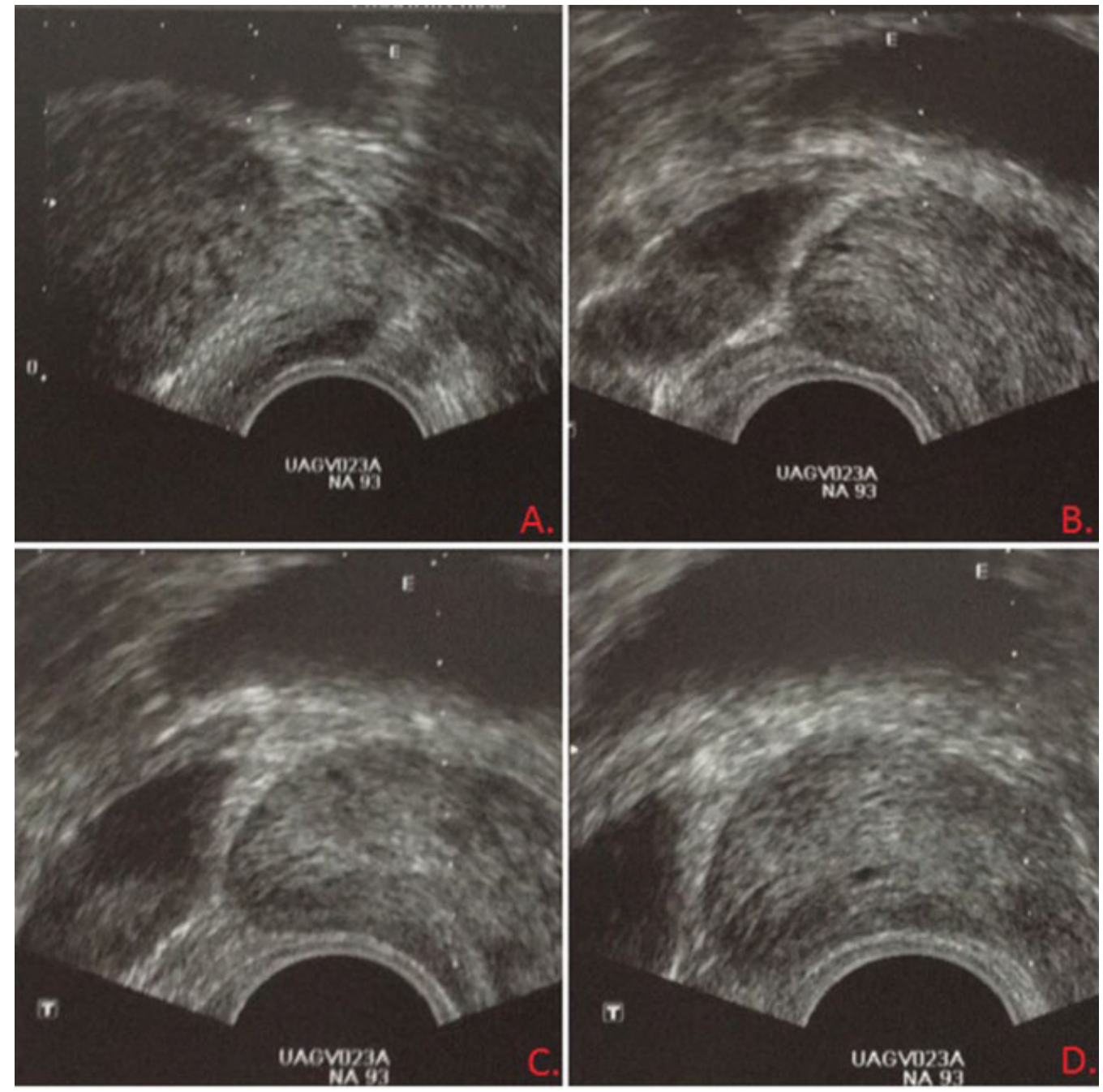

Fig. 4 (A) Plano transversal, biopsia de la base izquierda paramediana, el trazador (línea punteada) indica la trayectoria de la aguja de biopsia. (B, C y D) Plano longitudinal, Biopsia de la Base, el tercio medio y el ápex respectivamente, el trazador indica la trayectoria de la aguja de biopsia. (Original).

cualquier paciente llevado a BTRP que consultó por fiebre, debe sospechársele bacteremia. La segunda complicación más frecuente es el sangrado con una tasa de hasta un $19 \%$ en la serie de Nam y col., con una incidencia promedio de $1 \%$ a $4 \%$. La próstata secreta activador tisular del plasminógeno (tPA) y citrato que son anticoagulantes naturales y son los responsables del sangrado durante ese procedimiento. Djavan y Raajimakers y col. encontraron que entre un $23 \%$ y un $63 \%$ de los pacientes que se someten a BTRP por sextantes, pueden tener hematuria, el sangrado rectal no es infrecuente y se presenta en el 2,1\% y el 21,7\% de los pacientes, sin embargo, usualmente es de fácil control aplicando presión directa con el transductor o presion digital, incluso puede ser necesario otras estrategias como el taponamiento rectal con un condón inflado, anoscopía, sutura de la mucosa rectal, colonoscopia con infiltración de epinefrina o polidocanol y en casos muy severos, angiografía con embolización selectiva. Entre el 9,8\% y el 50,4\% pueden presentar hematospermia con una duración media de $4 \pm 1,4$ semanas, dicha situación genera ansiedad, recalcando la importantcia de explicar eso a los pacientes, especialmente que no tiene mayor importancia clínica. ${ }^{18,26,30}$

\section{Analgesia}

La BTRP es un procedimiento doloroso debido a que implica múltiples punciones con aguja de biopsia de 16-18 gauge. De todos los métodos evaluados, el más eficaz para el control del dolor durante el procedimiento, es el bloqueo del plexo nervioso periprostático guiado por ecografía. Históricamente, se realizaba con poca preocupación acerca del dolor experimentado por el paciente, debido a que el recto y la próstata se consideraban órganos con bajo umbral de sensibilidad y el procedimiento era bien tolerado por el paciente, inclusive hoy en día, el 33\% de los urólogos en los Estados Unidos, no utilizan ninguna técnica anestésica para llevar a cabo el procedimiento; se han descrito factores asociados al control del dolor; ansiedad por el procedimiento, antecedente de BTRP, y el número de cilindros obtenidos. El ápice de la próstata es la zona más dolorosa para la realización de la biopsia debido a que la mucosa ano-rectal se encuentra muy bien inervada por debajo de la línea dentada. ${ }^{32}$

Los sitios de infiltración para el bloqueo periprostático son determinados por la ubicación del paquete neurovascular y sus ramas que inervan la glándula. Para obtener un bloqueo 


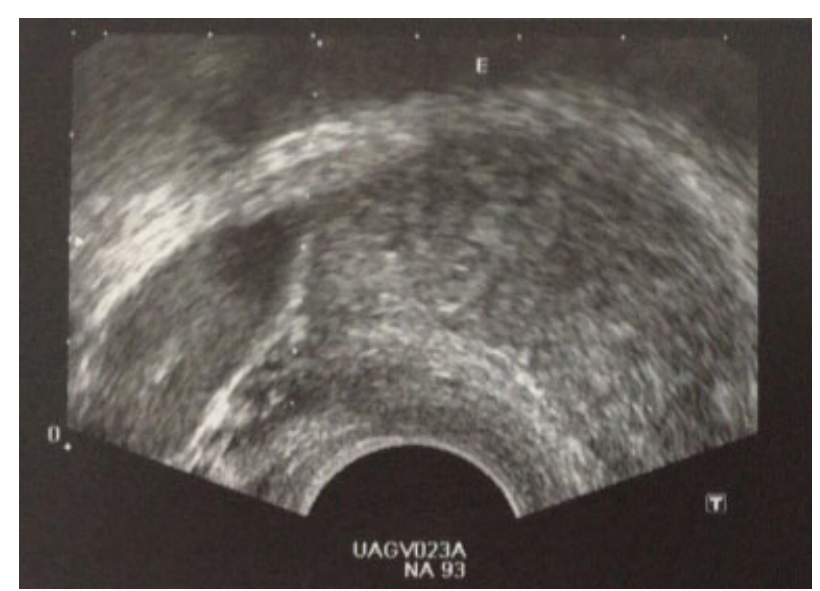

Fig. 5 Se observa el trazador (Línea punteada) en el ángulo vesiculoprostatico izquierdo y la infiltración de $5 \mathrm{~mL}$ de lidocaína al 1\% que se aprecia como una zona hipoecoica adyacente al ángulo vesiculoprostatico. (Original).

óptimo se debe infiltrar la base, el ápex y la cara lateral en cada lado de la próstata. ${ }^{32}$ Según la Canadian Urological Association (CUA), se debe realizar con una aguja peridural de $17 \mathrm{~cm}$ y 22gauge, el anestésico local que recomiendan es lidocaína sin epinefrina al 1 o $2 \%$. El esquema de infiltración más popular se realiza infiltrando $5 \mathrm{~mL}$ de lidocaína bilateralmente en la zona posterolateral donde se ubica el paquete neurovascular, en la base de la próstata justo lateral a la base de implantación de las vesículas seminales. $^{25}$ Otra alternativa es el bloqueo intraprostático, que se realiza en la zona periférica de la próstata y ha mostrado que en conjunto con el bloqueo periprostático hay mejor control del dolor y tolerancia del procedimiento. Se debe utilizar lidocaína en jalea como lubricante para permitir avanzar el transductor y lograr anestesia de la región perianal; en algunos casos seleccionados se puede infiltrar la piel y el tejido celular subcutáneo de la región perianal para manejo adicional del dolor causado por la inserción del transductor ${ }^{18,32}$ (- Fig. 5).

\section{Profilaxis Antibiótica}

La profilaxis antibiótica para microorganismos gram negativos es obligatoria en todos los pacientes que van a ser llevados a ese procedimiento $^{18,25}$ En el 2011, la revisión de Cochrane demostró una reducción de bacteriuria, bacteremia, fiebre, infecciones del tracto urinario y tiempo de hospitalización en pacientes que recibieron profilaxis en comparación con el grupo control. ${ }^{16}$ Las fluoroquinolonas son los antibióticos frecuentemente más utilizados como profilaxis antibiótica debido a que cubren enterobacterias y tienen buena disponibilidad en el parénquima prostático. ${ }^{33}$ Se han descrito cuatro factores de riesgo para E. Coli resistente a fluoroquinolonas: diabetes mellitus, exposición previa a fluoroquinolonas, hospitalización previa y cultivos previos con bacilos gram negativos resistente a fluoroquinolonas. Esos factores de riesgo son útiles para predecir el riesgo post biopsia de presentar infección por esos microorganismos. Se ha sugerido tomar un cultivo o hisopado rectal previo al procedimiento en los pacientes con factores de riesgo para E. Coli multirresistente y así iniciar profilaxis antibiótica con base en el perfil de sensibilidad del antibiograma. ${ }^{34}$

Existen tres abordajes a la hora de escoger el régimen de profilaxis: la monoterapia, la profilaxis aumentada y la profilaxis germen específica. La monoterapia consiste solo en el empleo de fluoroquinolonas para evitar la infección del tracto urinario, el aumento en la resistencia a esa clase de antibióticos ha obligado a buscar otras alternativas pero falta evidencia para usar otros antibióticos como la monoterapia, sin embargo, se ha demostrado que la piperacilina-tazobactam y la ceftriaxona son igual de efectivas a las fluoroquinolonas. La profilaxis aumentada se ha propuesto como una alternativa a la monoterapia bajo la creencia de que al dar profilaxis dual o monoterapia con un antibiótico de amplio espectro disminuyen las tasas de IVU, urosepsis y hospitalizaciones como demostró Yang y col., en su meta-análisis. Se han evaluado esquemas duales con Fluoroquinolonas y una dosis única de aminoglucósidos específicamente amikacina y gentamicina y el uso de una dosis de un carbapenémico como profilaxis aumentada, mostrando mejores resultados que la monoterapia pero con el riesgo de inducir mecanismos de resistencia antibiótica. En Colombia no se recomienda utilizar esquemas aumentados de profilaxis con carbapenémicos. El estudio de Castelblanco y col., en la Fundacion Santa Fe de Bogotá, incluyó 554 pacientes sometidos BTRP a quienes se les realizó hisopado rectal para determinar la resistencia a fluroquinolonas, y encontraron que el $45,8 \%$ de los pacientes, tenian microorganismos resistentes a las fluoroquinolonas y de ellos, el $60 \%$, habia recibido tratamiento con ciprofloxacina previo a la biopsia. $^{35}$ Más recientemente, el Michigan Urological Surgery Improvement Collaborative (MUSIC), es un consorcio que pretende mejorar la calidad y la costo efectividad del tratamiento de pacientes con cáncer de próstata a la vez que disminuir las complicaciones derivadas de la BTRP, uno de los estudios realizados con la base de datos de MUSIC, encontró que la tasa de ingreso a urgencias 30 días posterior a una BTRP es de $0,97 \%$ y el $95 \%$ de las admisiones fueron por complicaciones infecciosas. La mayoría de los microorganismos eran resistentes a las fluoroquinolonas, por lo que decidieron publicar una guía con dos esquemas de profilaxis antibiótica para BTRP. La guía del MUSIC propone que siempre se debe solicitar un hisopado rectal en todos los pacientes que seran llevados BTRP y proponen dos alternativas, la primera es profilaxis antibiótica germen especifica (hisopado rectal), y la segunda cuando no hay disponible un cultivo o hisopado rectal, se debe utilizar una profilaxis antibiótica aumentada con el fin de disminuir las complicaciones infecciosas derivadas de la BTRP ${ }^{36}$ (- Tabla 1).

A pesar de las recomendaciones del consorcio MUSIC, algunos estudios han demostrado que la profilaxis dirigida al perfil microbiológico obtenido en el cultivo rectal, no ha mostrado una reducción en las complicaciones de tipo infecciosas, eso debido a que la presencia de microorganismos resistentes a las quinolonas, no se traduce en infección en la mayoría de los casos, por lo que no se recomienda de rutina en todos los pacientes, excepto en paciente con riesgo de colonización por E. coli multirresistente, donde sí ha mostrado beneficio. ${ }^{33,37}$ 
Tabla 1 Profilaxis antibiótica germen-especifica \& profilaxis antibiótica aumentada

\begin{tabular}{|c|c|c|}
\hline \multicolumn{3}{|c|}{ Profilaxis antibiótica germen-especifica (hisopado rectal) } \\
\hline $\begin{array}{l}\text { Cultivo Sensible a } \\
\text { Fluoroquinolonas }\end{array}$ & $\begin{array}{l}\text { Cultivo Resistente a Fluoroquinolonas/ } \\
\text { Sensible a TMP/SMX o Cefalosporinas }\end{array}$ & $\begin{array}{l}\text { Cultivo Resistente a Fluoroquinolonas, } \\
\text { TMP/SMX y Cefalosporinas }\end{array}$ \\
\hline $\begin{array}{l}\text { Ciprofloxacina } 500 \mathrm{mg} \\
\text { ( } 1 \text { Tableta } 1 \text { hora antes de la BTRP y la } \\
\text { noche después) } \\
\text { o } \\
\text { Levofloxacina } 750 \mathrm{mg} \text { (Dosis única, } \\
60 \text { minutos antes) }\end{array}$ & $\begin{array}{l}\text { TMP/SMX DS (VO) } \\
\text { ( } 1 \text { tableta } 1 \text { hora antes de la BTRP y la } \\
\text { noche después) } \\
\text { o } \\
\text { Cefazolina IM } 1 \text { gramo ( } 1 \text { hora antes) } \\
\text { o } \\
\text { Ceftriaxona IM } 1 \text { gramo ( } 1 \text { hora antes) }\end{array}$ & $\begin{array}{l}\text { Gentamicina IM/IV 120/160 mg } \\
\text { (30/60 minutos antes) } \\
\text { o } \\
\text { Fosfomicina VO } 3 \mathrm{gr} \\
\text { (La noche anterior a la BTRP) }\end{array}$ \\
\hline \multicolumn{3}{|c|}{ Profilaxis antibiótica aumentada (no hay disponible hisopado rectal o factores de riesgo) } \\
\hline Profilaxis de elección & Alternativas & $\begin{array}{l}\text { Alergia a penicilinas, fluoroquinolonas o } \\
\text { cefalosporinas }\end{array}$ \\
\hline $\begin{array}{l}\text { Ciprofloxacina/Levofloxacina } \\
+ \\
\text { Gentamicina IM }\end{array}$ & $\begin{array}{l}\text { Ciprofloxacina/Levofloxacina } \\
\text { o } \\
\text { TMP/SMX + Cefazolina IM } \\
\text { o } \\
\text { Cefuroxime }\end{array}$ & $\begin{array}{l}\text { Gentamicina IM/IV } \\
\text { o } \\
\text { TMP/SMX (VO) + Clindamicina (IM) } \\
\text { o } \\
\text { Fosfomicina (VO) }\end{array}$ \\
\hline
\end{tabular}

Abreviaturas: DS, Double Strength TMP/SMX 160/800 mg; IM, Intramuscular; VO, Vía oral.

${ }^{*}$ Se recomienda administrar antibioticos IV cuando: BLEE + en hisopado rectal, Antecedente de infección post-biopsia por microorganismos resistentes a fluoroquinolonas. Antecedente de prostatitis que no responde a fluoroquinolonas o que ha recibido múltiples ciclos de fluoroquinolonas.

El aumento de la resistencia antibiótica ha generado gran interés en buscar técnicas que puedan minimizar el riesgo de infección, incluye preparación pre-biopsia con enemas, el número de cilindros tomados y la técnica de desinfección de la aguja de biopsia. La preparación pre-biopsia con enema usando fosfato de sodio o solución salina (enema jabonoso), no demostró efecto protector, en contraste el enema con yodopovidona ha demostrado reducir las infecciones postbiopsia. Estudios recientes indican que la biopsia transperineal de la próstata puede tener tasas de detección de cáncer comparables con el abordaje transrectal, con una menor incidencia en la tasa de infecciones. Grummet y col. describieron una tasa de $0,076 \%$ de sepsis en 6609 pacientes que fueron llevados a biopsia transperineal. La desinfección de la aguja con formol ha demostrado ser costo-efectiva para reducir las complicaciones por infecciones, Issa y col. sugiere que la desinfección con formol al 10\% después de cada disparo, puede minimizar el riesgo de infección post-biopsia. ${ }^{37}$

\section{Anticoagulación-antiagregación}

En una de las guías de práctica clínica de la CUA, se recomienda suspender los agentes antiagregantes 7-14 días antes de realizar el procedimiento. Los anticoagulantes se deben retirar 4-5 días antes de la biopsia, ${ }^{25}$ sin embargo, el ácido acetil salicílico (ASA), en dosis bajas, no necesita ser suspendido. ${ }^{18}$ Se debe evaluar la indicación de anticoagulación y el riesgobeneficio de suspenderla, individualizando a cada paciente; en los pacientes anticoagulados se debe llevar el INR a 1,5 para poder realizar la BTRP, en los que tienen riesgo elevado de presentar un evento tromboembólico, se recomienda hacer terapia puente con heparina no fraccionada o de bajo peso molecular para suspender los cumarinicos. ${ }^{18}$ Los inhibidores del factor Xa y la trombina: apixaban, rivaroxaban y dabigatran respectivamente, deben suspenderse 2-5 días antes del procedimiento sin necesidad de hacer terapia puente a excepción del rivaroxaban en el que sí se debe hacer por el riesgo elevado de enfermedad cerebrovascular. ${ }^{18,25}$

\section{Enema}

Un 80\% de los urólogos indican la realización de un enema como parte de la preparación para la biopsia, debido a que puede ayudar a mejorar la ventana acústica lo que se traduce en mayor calidad de la imagen. En cuanto al uso de enemas en la prevención de infecciones no contamos con evidencia de buena calidad para recomendar o no su uso. ${ }^{18,25,28,33} \mathrm{El}$ uso de los enemas con yodopovidona se ha popularizado en norteamérica, al punto que la American Urological Association (AUA), lo deja a discreción del médico tratante, mientras que en las guías europeas, no está recomendado su uso de rutina. Park y col. evaluó el uso rectal de supositorios de yodopovidona encontrando una reducción en el número de colonias bacterianas. Las limitaciones del uso de yodopovidona como antiséptico rectal son que la polivinilpirrolidona que hace parte de ese yodóforo, actúa en un medio seco, sin embargo, el recto es una cavidad húmeda que contiene materia fecal y moco. La efectividad de la clorhexidina no se ve afectada por los factores mencionados anteriormente y tiene una alta biodisponibilidad en piel y mucosas, no obstante, no hay estudios que comparen la eficacia del enema con clorhexidina con el de yodopovidona en el contexto de la BTRP. ${ }^{33}$

\section{Re- Biopsia: Biopsia por saturación y Biopsia guiada por RNMmp}

En nuestra práctica clínica es común enfrentarnos con el dilema de si debemos biopsiar nuevamente a un paciente en 
el contexto de un PSA persistentemente elevado y una BTRP negativa. Las guías de la NCCN recomiendan que de persistir la sospecha clínica de cáncer de próstata en un paciente con una BTRP negativa, debe considerarse la biopsia por saturación o la biopsia guiada por RNMmp, eso basado en la premisa de que con las biopsias por saturación, aumenta la tasa de detección por vía transrectal hasta $31,4 \%$, transperineal $25,7 \%$, y guiada por resonancia entre el $41 \%$ y el $56 \%{ }^{38,39}$

Se han descrito varias estrategias para asesorar mejor a los pacientes antes de una re-biopsia, entre ellas se encuentra la densidad del PSA (dPSA), velocidad del PSA (vPSA), y porcentaje de PSA libre (\%PSA). Una condición para poder utilizar esos parámetros es que el nivel de PSA debe estar entre 4 y $10 \mathrm{ng} / \mathrm{mL}$ para no afectar el valor predictivo de los mismos. La dPSA es el resultado de dividir el nivel de PSA sobre la volumetría prostática calculada por USTR, entre menor sea más sugestivo de hiperplasia prostática benigna, el punto de corte es $0,15 \mathrm{ng} / \mathrm{mL} / \mathrm{cc}$. La vPSA se debe determinar con 3 valores diferentes de PSA tomados en un intervalo de tiempo de 18 meses, se han estudiado varios puntos de corte, sin embargo, el que ha mostrado mayor rendimiento es una vPSA $>0,35 \mathrm{ng} / \mathrm{mL} /$ año de acuerdo con el estudio de Carter y col. El \%fPSA es la proporción entre el PSA libre/PSA total (tPSA), y esa medición se basa en la premisa que el $60 \%$ al $90 \%$ del PSA circula unido a alfa-1 antiquimiotripsina, dicha proporción en hombres con cáncer de próstata tiende a ser menor. El punto de corte que mostró mayor tasa de detección de cáncer (95\%) es 25\%, sin embargo, hay estudios que aceptan un punto de corte de hasta el $15 \%$.

El PCA3 es un marcador urinario, en el que se identifica una cadena RNA específico del tejido prostático, el cual fue aprobado por la FDA en el contexto de los pacientes con BTRP negativa y sospecha clínica de cáncer. Tiene una sensibilidad del $78 \%$, a expensas de una especificidad del $57 \%$ y un valor predictivo positivo de un $34 \%{ }^{4,40}$ La combinación de diferentes isoformas e índices del PSA como el Prostate Health Index (PHI) Score, el cual combina el tPSA, fPSA y proPSA o el 4Kscore que combina el tPSA, fPSA, PSA intacto y la calicreína humana de tipo 2 (hK2), han mostrado un buen desempeño a la hora de evaluar la necesidad de re-biopsia, ${ }^{40}$ aunque su disponibilidad es limitada en Colombia.

\section{Biopsia por Saturación}

La biopsia por saturación, a pesar del advenimiento del uso de RNMmp para evaluar a los hombres con biopsia negativa y como guía para una nueva biopsia, figura aun en las recomendaciones de las guías de práctica clínica actuales, como una estrategia que ofrece una tasa de detección de cáncer superior a la de la BTRP de doble sextante, pero inferior a la BTRP guiada por RNMmp. Debe reservarse únicamente en hombres con una BTRP negativa, ya que no ha mostrado superioridad en el contexto de la primera biopsia, con respecto a la detección de cáncer de próstata. La biopsia por saturación implica tomar entre 18 y 24 cilindros de manera sistemática, incluyendo la zona transicional y el estroma fibromuscular anterior. Hay dos abordajes para la biopsia por saturación, la vía transrectal o transperineal,
La ventaja del abordaje transrectal es que se puede realizar en el consultorio y el urólogo se encuentra más familiarizado con la US transrectal, sin embargo, a expensas de una tasa de detección de cáncer de un 31\%, mayor riesgo de infección, bacteriemia post-biopsia y sangrado. La biopsia transperineal por saturacón ha mostrado menores tasas de infección, es más tolerable por el paciente, y se consideró el patrón de oro en el estudio (PROMIS) con una sensibilidad del 95\% y una tasa de detección de cáncer de un 39\% a un 55\% sin embargo, esa modalidad no reemplaza la BTRP por saturación en el contexto de re-biopsia y se deja a discreción del urólogo la vía de abordaje.

\section{Biopsia Guiada por RNMmp}

La RNM se utiliza desde 1990 para evaluar pacientes con cáncer de próstata, inicialmente para evaluar extensión extracapsular y compromiso de las vesículas seminales. El uso de antenas endorectales, el desarrollo tecnológico de imanes de gran campo que disminuye la relación señal/ruido y el advenimiento de RNMmp, ha puesto a esa prueba diagnóstica como el examen de elección en el paciente con una BTRP negativa. ${ }^{2}$ La RNMmp utiliza las secuencias Dynamic contrast enhanced (DCE), que evalúa la perfusión de la próstata mediante el paso del gadolinio por la glándula, Difussion weighted imaging (DWI), que mide el movimiento browniano de las moléculas de agua en la próstata, por lo que los tejidos con alta celularidad tienen un coeficiente de difusión bajo, útil en la caracterización de lesiones tumorales; ;,41-44

El examen diagnostico ideal en cáncer de próstata, debe ser mínimamente invasivo, tener pocos eventos adversos, identificar un alto porcentaje de CPCS y evitar la sobredetección de cáncer no significativo. Con la estandarizacion del reporte de la RNMmp y la universalidad de ese estudio, se ha propuesto como parte de la evaluación inicial del paciente con sospecha de cáncer de próstata. Se evaluó en un estudio prospectivo multicéntrico de cohorte pareada (PROMIS) la RNMmp como parte de la evaluacion inicial en el paciente con sospecha de cáncer de próstata en el que concluyeron que se puede recomendar la RNMmp como estudio inicial a todos los hombres con sospecha de cáncer antes de la BTRP. ${ }^{42,43}$ La limitacion de ese estudio fue que por el enmascaramiento no se biopsiaron lesiones sospechosas y no se pudo establecer la efectividad de las biopsias guiadas por mpMRI, duda que se resolvió con el estudio Prostate Evaluation for Clinically Important Disease: Sampling Using Image Guidance or Not? (PRECISION), un estudio multicentrico, aleatorizado, y de no inferioridad, en el que se comparó la biopsia dirigida con RNMmp con la BTRP estándar y encontraron que con la RNMmp se evita en un $25 \%$ de pacientes una biopsia innecesaria, la tasa de detección de CPCS es de un 39\% comparado con un $27 \%$ con la tecnica estándar y la detección de cáncer clínicamente significativo fue de un $9 \%$ vs $22 \%$ en la BTRP ecodirigida, con un menor número de eventos adversos en el brazo de biopsia dirigida por RNMmp, dado a que se toma un menor número de cilindros. Por lo que en algunos países, ya se ha adoptado la RNMmp de entrada como el estándar de manejo. ${ }^{45}$ 
Hay tres técnicas para guiar la biopsia por RNM. La primera, es la técnica fusión-cognitiva, consiste en calcular la localización de la lesión evidenciada por RNM y luego mentalmente trasladarla al objetivo en la imagen obtenida durante la US transrectal. ${ }^{41-44}$ La segunda técnica es la biopsia In-Bore, que es realizada con el paciente en el resonador donde se visualiza la lesión con una RNM en tiempo real para corroborar la localización de la aguja. ${ }^{41}$ Por último, está la biopsia fusión guiada con RNM/US en tiempo real. En ese método, la imagen de la próstata es reconstruida en 3-D con los objetivos de la biopsia, gracias a la fusión de la imagen por ecografía y la RNMmp y así poder dirigir los movimientos del transductor y la pistola de biopsia. ${ }^{43,44}$ Aún no se ha establecido cuál de los tres abordajes es superior y se continúa recomendando la biopsia fusión cognitiva en el escenario de la re-biopsia, sin embargo, se ha establecido que para lesiones pequeñas o localizadas en sitios de difícil acceso como la porción apical o anterior de la glándula, los métodos por fusión o in-bore tienen mejor desempeño en comparación con la biopsia fusión-cognitiva. ${ }^{43}$

\section{Biopsia del Lecho Prostático}

Entre el 27\% y el 53\% de los pacientes que son llevados a prostatectomía radical o radioterapia, tienen recaida bioquimica. ${ }^{46}$ La biopsia del lecho es un procedimiento donde se toman minimo 4 muestras de la anastomosis uretrovesical (dos de cada lado), cuyo objetivo es confirmar una recurrencia local, ya que se ha encontrado que un resultado positivo influye sobre los desenlaces de la radioterapia de salvamento. ${ }^{47}$ Naya y col. evaluaron 100 pacientes que fueron sometidos a biopsia del lecho prostático y concluyeron que solo se debe biopsiar pacientes con PSA mayor a $0,5 \mathrm{ng} / \mathrm{mL}$, examen rectal anormal o USTR con hallazgos anormales. ${ }^{48}$ Deliveliotis y col. estudiaron 30 pacientes con recaída bioquímica posterior a prostatectomía radical, quienes fueron llevados a biopsia de la anastomosis uretrovesical, documentando recaida locoregional en el $40 \%$ de los casos, recomendando que aquellos pacientes con recaída bioquímica y ausencia de metástasis en los estudios de extension (Gammagrafia osea y TAC toraco-abdominal), deben ser llevados a biopsia de lecho prostático. $^{49}$

En la actualidad es controversial si se debe hacer biopsia del lecho. En las guías de práctica clínica, la guía de cáncer de próstata del 2017 de la NCCN, sugiere realizar la biopsia del lecho cuando el PSA sigue siendo detectable (persistencia del PSA), o cuando el PSA es negativo después de la PR y se eleva eventualmente (recurrencia del PSA), sin embargo, debe realizarse espcialmente si hay imágenes que sugieren la recaida local. ${ }^{50}$ Por el contrario, la guía del National Institute for Health and Care Excellence (NICE), no recomienda la biopsia del lecho prostático. ${ }^{51}$ Al igual que la guia de práctica clínica de la European Association of Urology (EAU), no recomiendan ese procedimiento como parte del estudio de la recaida bioquimica, debido a que la biopsia del lecho presenta una sensibilidad del $40-71 \%$ con un PSA $>1 \mathrm{ng} / \mathrm{mL}$ y del $14-45 \%$ con un PSA $<1 \mathrm{ng} / \mathrm{mL}$, valores relativamente bajos, por lo cual se recomienda iniciar radioterapia de salvamento sin necesidad de confirmación histopatológica de la recurrencia. ${ }^{46}$

\section{Consejos y trucos de nuestra práctica clínica}

En nuestra práctica clínica realizamos aproximadamente 1100 BTRP anuales y la siguiente es nuestra guía de consejos y trucos con la que hemos encontrado los mejores resultados y la menor tasa de complicaciones.

1. Se aplica la tabla de factores de riesgo para infección post biopsia de la AUA, se toma muestra para cultivo/hisopado rectal y urocultivo con el fin de individualizar la profilaxis en pacientes sin bacteriuria asociada a catéter. En pacientes sin factores de riesgo y con sensibilidad a las quinolonas en la flora rectal, se administra ciprofloxacina oral. Si el paciente tiene factores de riesgo, con flora rectal sensible, función renal normal, se administra profilaxis antibiótica aumentada con una fluoroquinolona asociada a aminoglucósido o ceftriaxona o fosfomicina. Si hay flora rectal resistente, independiente a factores de riesgo, se administra fosfomicina. En caso de aparición de gérmenes BLEE en el recto se indica profilaxis con fosfomicina o carbapenémicos. En pacientes con bacteriuria, se ajusta el manejo antibiótico al germen.

2. Se aplican enemas fosfato-bifosfato de $133 \mathrm{~mL}$ intrarectal (mejora la ventana acústica), la noche anterior y 3 horas antes del procedimiento. Puede adicionarse a nivel intrarectal, con el fin de disminuir la carga bacteriana, supositorios de iodopovidona o enemas con iodopovidona.

3. Con respecto a la anticoagulación/antiagregación, recomendamos suspender antiagregantes 7 días (en caso de uso para prevención primaria), antes de realizar el procedimiento. El ácido acetil salicílico (ASA) en dosis bajas no necesita ser suspendido. Se debe suspender la enoxaparina como tromboprofilaxis 12 horas antes del procedimiento y como anticoagulación plena 24 horas antes, los cumarinicos se deben suspender 3-4 días antes y se debe llevar el INR a 1,5 para poder realizar la BTRP, en los que tienen riesgo elevado de presentar un evento tromboembólico, se recomienda hacer terapia puente con heparina no fraccionada o de bajo peso molecular. Los inhibidores del factor Xa y la trombina, apixaban, rivaroxaban $\mathrm{y}$ dabigatran respectivamente, deben suspenderse 2-5 días antes del procedimiento sin necesidad de hacer terapia puente a excepción del rivaroxaban en el que sí se debe hacer por el riesgo elevado de enfermedad cerebrovascular.

4. Utilizamos lidocaína en jalea para lubricación del canal rectal y como anestésico local, se introduce la eco-sonda la cual utiliza un transductor mono o biplanar (End-fire, Side-fire) y se realiza una exploración ecográfica hasta delimitar la anatomía de la próstata, vejiga y vesículas seminales. Con una aguja peridural de $17 \mathrm{~cm}$ y 22 -gauge, se infiltra $5 \mathrm{~mL}$ lidocaína sin epinefrina al $1 \%$ bilateralmente en el ángulo vesiculoprostático, y en ocasiones una infiltración intraprostática. 
5. Se calcula la volumetría prostática utilizando la fórmula del elipsoide, se toman las medidas de largo y ancho en un plano transversal y la medida de altura en un plano longitudinal.

6. Se introduce la aguja de biopsia a través del canal de trabajo/guia del transductor y se toman las muestras inicialmente del lado derecho de la próstata, siguiendo el esquema en doble sextante, se rotulan los cilindros lateral y paramediano de la base derecha como 1 y 2, los cilindros mediano y paramediano del tercio medio derecho 3 y 4, los cilindros mediano y paramediano del ápex derecho 5 y 6 y se envían a patología en un frasco con formol al $10 \%$ diluido en solución salina los dos cilindros, se repite el mismo proceso en el lado contralateral siguiendo la numeración de 7 a 12 . Se puede realizar la toma en el plano longitudinal o transversal, especialmente cuando se requiere tomar muestras muy laterales de la zona periférica.

7. Al finalizar se realiza compresión de la pared rectal y la próstata con el transductor durante 2 minutos con el fin de mejorar el control hemostático.

\section{Conclusiones}

La BTRP ecodirigida es el estándar de cuidado actual en los pacientes con sospecha de cáncer de próstata; se realizan más de un millón de BTRP anualmente en los Estados Unidos, por lo que se han hecho grandes esfuerzos para mejorar su desempeño diagnostico y disminuir las complicaciones asociadas al procedimiento. En nuestro estudio presentamos las recomendaciones actuales después de hacer una revisión exhaustiva de la literatura con la idea de que el lector puede familiarizarse con los esquemas, instrumentos y técnicas utilizadas en BTRP, así como las posibles complicaciones y las estrategias para prevenirlas y concluimos, con nuestros consejos y trucos, que han sido adaptadas a la realidad de nuestro país y han mostrado un muy buen desempeño diagnóstico asociado a una baja tasa de complicaciones permitiéndonos, con tranquilidad, realizar mas de 1000 BTRP anuales.

\section{Bibliografía}

1 Siegel RL, Miller KD, Jemal A. Cancer statistics, 2016. CA Cancer J Clin 2016;66(01):7-30

2 Logan JK, Rais-Bahrami S, Turkbey B, et al. Current status of magnetic resonance imaging (MRI) and ultrasonography fusion software platforms for guidance of prostate biopsies. BJU Int 2014;114(05):641-652

3 Howlander N. (2004). SEER Cancer Statistics Review 1975-2004. http://seer.cáncer.gov/csr/1975_2004/

4 Carroll PR, Parsons JK, Andriole G, et al. NCCN guidelines insights: prostate cáncer early detection, version 2.2017. J Natl Compr Canc Netw 2016;14(05):509-519

5 Vickers AJ, Cronin AM, Björk T, et al. Prostate specific antigen concentration at age 60 and death or metastasis from prostate cancer: case-control study. BMJ 2010;341:c4521

6 Randazzo M, Müller A, Carlsson S, et al. A positive family history as a risk factor for prostate cancer in a population-based study with organised prostate-specific antigen screening: results of the Swiss European Randomised Study of Screening for Prostate Cancer (ERSPC, Aarau). BJU Int 2016;117(04):576-583
7 Barocas DA, Grubb R III, Black A, et al. Association between race and follow-up diagnostic care after a positive prostate cancer screening test in the prostate, lung, colorectal, and ovarian cancer screening trial. Cancer 2013;119(12):2223-2229

8 Presti JC Jr, O’Dowd GJ, Miller MC, Mattu R, Veltri RW. Extended peripheral zone biopsy schemes increase cancer detection rates and minimize variance in prostate specific antigen and age related cancer rates: results of a community multi-practice study. J Urol 2003;169(01):125-129

9 Ukimura O, Coleman JA, de la Taille A, et al. Contemporary role of systematic prostate biopsies: indications, techniques, and implications for patient care. Eur Urol 2013;63(02):214-230

10 Schoots IG, Roobol MJ, Nieboer D, Bangma CH, Steyerberg EW, Hunink MG. Magnetic resonance imaging-targeted biopsy may enhance the diagnostic accuracy of significant prostate cancer detection compared to standard transrectal ultrasound-guided biopsy: a systematic review and meta-analysis. Eur Urol 2015;68(03):438-450

11 Taira AV, Merrick GS, Galbreath RW, et al. Performance of transperineal template-guided mapping biopsy in detecting prostate cancer in the initial and repeat biopsy setting. Prostate Cancer Prostatic Dis 2010;13(01):71-77

12 Yeo L, Patel D, Bach C, et al. (2011). The development of the modern prostate biopsy. In Prostate Biopsy. InTech

13 Herranz Amo F, Diéz Cordero JM, Cabello Benavente R. Evolución de la técnica de biopsia transrectal ecodirigida de la próstata. Arch Esp Urol 2006;59(04):385-396

14 Aarnink RG, Beerlage HP, De La Rosette JJ, Debruyne FM, Wijkstra $\mathrm{H}$. Transrectal ultrasound of the prostate: innovations and future applications. J Urol 1998;159(05):1568-1579

15 Hodge KK, McNeal JE, Terris MK, Stamey TA. Random systematic versus directed ultrasound guided transrectal core biopsies of the prostate. J Urol 1989b142(01):71-74, discussion 74-75

16 Levine MA, Ittman M, Melamed J, Lepor H. Two consecutive sets of transrectal ultrasound guided sextant biopsies of the prostate for the detection of prostate cancer. J Urol 1998;159(02):471-475, discussion 475-476

17 Mitterberger M, Horninger W, Aigner F, et al. Ultrasound of the prostate. Cancer Imaging 2010;10(01):40-48

18 Wein AJ, Kavoussi LR, Campbell MF. 1. (2015). Campbell-Walsh urology (11th ed.). Philadelphia, PA: Elsevier Saunders

19 Patel U, Rickards D. (2002). Handbook of transrectal ultrasound and biopsy of the prostate. Taylor \& Francis

20 Rodriguez E Jr, Skarecky D, Narula N, Ahlering TE. Prostate volume estimation using the ellipsoid formula consistently underestimates actual gland size. J Urol 2008;179(02):501-503

21 MacMahon PJ, Kennedy A-M, Murphy DT, Maher M, McNicholas MM. Modified prostate volume algorithm improves transrectal US volume estimation in men presenting for prostate brachytherapy. Radiology 2009;250(01):273-280

22 Ching CB, Moussa AS, Li J, Lane BR, Zippe C, Jones JS. Does transrectal ultrasound probe configuration really matter? End fire versus side fire probe prostate cancer detection rates. J Urol 2009;181(05):2077-2082, discussion 2082-2083

23 Rojas AFG. Evaluación volumétrica de la próstata. Rev Urol Colombiana 2017;26(01):74-75

24 Kaye KW, Horwitz CA. Transrectal ultrasound-guided prostate biopsies using new automatic gun: analysis of 100 consecutive cases. J Endourol 1989;3(02):155-161

25 El-Hakim A, Moussa S. CUA guidelines on prostate biopsy methodology. Can Urol Assoc J 2010;4(02):89-94

26 Gonzalez C, et al. AUA/SUNA White Paper on the Incidence, Prevention and Treatment of Complications Related to Prostate Needle Biopsy

27 Stinchcomb S. AUA/Optimal Techniques of Prostate Biopsy and Specimen Handling (White paper)

28 Kam SC, Choi SM, Yoon S, et al. Complications of transrectal ultrasound-guided prostate biopsy: impact of prebiopsy enema. Korean J Urol 2014;55(11):732-736 
29 Chiang IN, Chang SJ, Pu YS, Huang KH, Yu HJ, Huang CY. Major complications and associated risk factors of transrectal ultrasound guided prostate needle biopsy: a retrospective study of 1875 cases in taiwan. J Formos Med Assoc 2007;106(11):929-934

30 Nam RK, Saskin R, Lee Y, et al. Increasing hospital admission rates for urological complications after transrectal ultrasound guided prostate biopsy. J Urol 2010;183(03):963-968

31 Loeb S, Vellekoop A, Ahmed HU, et al. Systematic review of complications of prostate biopsy. Eur Urol 2013;64(06):876-892

32 Nazir B. Pain during transrectal ultrasound-guided prostate biopsy and the role of periprostatic nerve block: what radiologists should know. Korean J Radiol 2014;15(05):543-553

33 Hwang EC, Jung SI, Seo YH, et al. Risk factors for and prophylactic effect of povidone-iodine rectal cleansing on infectious complications after prostate biopsy: a retrospective cohort study. Int Urol Nephrol 2015;47(04):595-601

34 Saade EA, Suwantarat N, Zabarsky TF, Wilson B, Donskey CJ. Fluoroquinolone-Resistant Escherichia coli Infections After Transrectal Biopsy of the Prostate in the Veterans Affairs Healthcare System. Pathog Immun 2016;1(02):243-257

35 Castelblanco DM, Gómez F, Cataño JG, Plata M, Trujillo CG. Cultivo rectal para detección de gérmenes resistentes a fluoroquinolonas y reducción de riesgo de bacteriemia en pacientes llevados a biopsia de próstata en un hospital de nivel IV de atención. Univ Méd 2015; 56(01):xx

36 Womble PR, Dixon MW, Linsell SM, et al; Michigan Urological Surgery Improvement Collaborative. Infection related hospitalizations after prostate biopsy in a statewide quality improvement collaborative. J Urol 2014;191(06):1787-1792

37 Walker JT, Singla N, Roehrborn CG. Reducing Infectious Complications Following Transrectal Ultrasound-guided Prostate Biopsy: A Systematic Review. Rev Urol 2016;18(02):73-89

38 Roethke M, Anastasiadis AG, Lichy M, et al. MRI-guided prostate biopsy detects clinically significant cancer: analysis of a cohort of 100 patients after previous negative TRUS biopsy. World J Urol 2012;30(02):213-218

39 Abdollah F, Novara G, Briganti A, et al. Trans-rectal versus transperineal saturation rebiopsy of the prostate: is there a difference in cancer detection rate? Urology 2011;77(04):921-925
40 National Comprehensive Cancer Network. Prostate early detection (Version 2.2016). https://www.nccn.org/professionals/physician_gls/ PDF/prostate_detection.pdf

41 Hamdy FC, Donovan JL, Lane JA, et al; ProtecT Study Group. 10 -year outcomes after monitoring, surgery, or radiotherapy for localized prostate cancer. N Engl J Med 2016;375(15): 1415-1424

42 Ahmed HU, El-Shater Bosaily A, Brown LC, et al; PROMIS study group. Diagnostic accuracy of multi-parametric MRI and TRUS biopsy in prostate cancer (PROMIS): a paired validating confirmatory study. Lancet 2017;389(10071):815-822

43 Rosenkrantz AB, Verma S, Choyke P, et al. Prostate magnetic resonance imaging and magnetic resonance imaging targeted biopsy in patients with a prior negative biopsy: a consensus statement by AUA and SAR. J Urol 2016;196(06):1613-1618

44 Marks L, Young S, Natarajan S. MRI-ultrasound fusion for guidance of targeted prostate biopsy. Curr Opin Urol 2013;23(01):43-50

45 Kasivisvanathan V, Rannikko AS, Borghi M, et al; PRECISION Study Group Collaborators. MRI-targeted or standard biopsy for prostate-cancer diagnosis. N Engl J Med 2018;378(19): 1767-1777

46 Mottet N, Bellmunt J, Bolla M, et al. EAU-ESTRO-SIOG Guidelines on Prostate Cancer. Part 1: Screening, Diagnosis, and Local Treatment with Curative Intent. Eur Urol 2017;71(04):618-629

47 Saleem MD, Sanders H, Abu El Naser M, El-Galley R. Factors predicting cancer detection in biopsy of the prostatic fossa after radical prostatectomy. Urology 1998;51(02):283-286

48 Naya Y, Okihara K, Evans RB, Babaian RJ. Efficacy of prostatic fossa biopsy in detecting local recurrence after radical prostatectomy. Urology 2005;66(02):350-355

49 Deliveliotis C, Manousakas T, Chrisofos M, Skolarikos A, Delis A, Dimopoulos C. Diagnostic efficacy of transrectal ultrasoundguided biopsy of the prostatic fossa in patients with rising PSA following radical prostatectomy. World J Urol 2007;25 (03):309-313

50 Prostate Cancer: Clinical Practice Guideline in Oncology. NCCN; 2017

51 Prostate Cancer: diagnosis and treatment. Commissioned by the National Institute for Health and Care Excellence; 2014 